\title{
Securing Personal Data using Block chain in Autonomous Vehicle
}

\author{
D. Prem Raja, V. Vasudevan
}

\begin{abstract}
Autonomous vehicles like Driverless cars are seen only in science fiction movies but in 2019 they are becoming a veracity and reality. People all around the world are excited to watch the driverless car in reality. Complete driverless car is still at an advanced testing stage. An autonomous vehicle promises to improve traffic safety while at the same time it must not be prone to hacking. Even though the existence of the autonomous car is in reality there is a possibility of hackers to hack the vehicle and retrieve the precious data. To stop this kind of hacking we propose a block chain technique that safe guards the data that is fed to the autonomous car during the manufacturing stage and this cannot be deleted without proper permission.
\end{abstract}

Keywords : Autonomous Vehicle, Block chain, Hackers

\section{INTRODUCTION} movies only, are now becoming an everyday reality. Autonomous vehicle is expected to considerably scale back collisions and enhance quality for the older and also the disabled and scale back the necessity for car parking zone within the cities. The autonomous vehicles are expected to play a key function in the future of city transportation systems, as they supply attainable for more safety, improved productivity, larger accessibility, remarkable impact on the surroundings. We see a sudden hype in the concept of autonomous cars, as industry leaders are on their way to redefining transportation. Autonomous Vehicles have won full-size attention from each academia and industry. The Autonomous Vehicles are a complex machine composed of many subsystems, making them a ordinary target for attackers. Therefore a firmware of special subsystems wanted to be up to date to the modern version by way of the manufacture to repair bugs and introduce new points like safety patches.

\section{RELATED WORK}

A firm ware update scheme based on block chain and smart contract is introduced for autonomous vehicles. A smart contract is used to make sure the authenticity and integrity of firm ware updates ${ }^{[1]}$. A UML model is predicted to find the nearest fuel / charging station for charging the autonomous car / the electric vehicle while the vehicle is in

Revised Manuscript Received on December 05, 2019

* Correspondence Author

Prem Raja D*, Department of information Technology, Kalasalingam Academy of Research and Education,Tamil Nadu, INDIA.

Vasudevan V, Department of information Technology, Kalasalingam Academy of Research and Education, Tamil Nadu, INDIA.
Self-driving vehicles, which once appeared in the sci-fi

the movement. The system checks for the nearest charging station and intimates it to the passenger or the operating officer $^{[2]}$.

Unmanned automobiles will play essential roles in our society (Fig.4). From handing over your mail to mapping an vicinity to unarming a bomb, they will grow to be essential. Video channels used for the manage of unmanned cars are of specific importance, having notable susceptibility to hijack, jamming and spoofing attacks ${ }^{[3]}$.

AMHL's can be realized from any (linear) homomorphic operation. A construction based on CDSA, thereby catering the vast majority of crypto currencies deployed today performance valuation shows that AMHLs are practical ${ }^{[4]}$. SDPSs, which intention to ensure the safety and privacy of sensor data, are the problem of an widespread body of literature (Fig.1). In particular, the IS community has made vast effort to look at problems of protection and privacy which has resulted in a variety of design theories ${ }^{[5]}$.
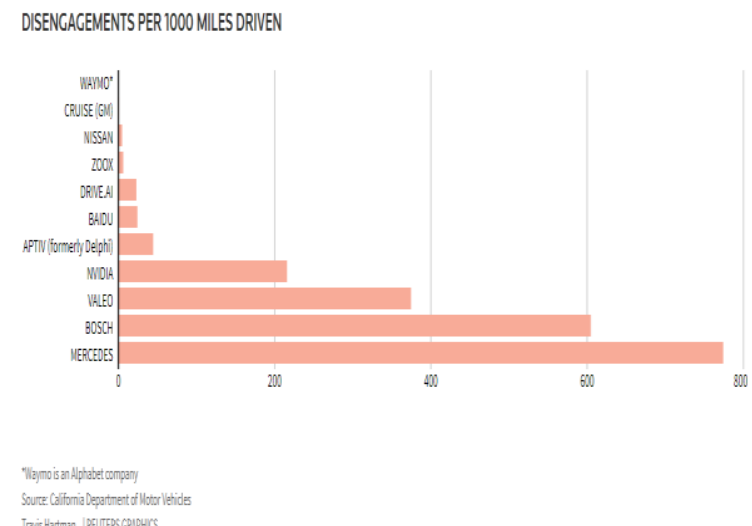

Fig.1:Disengagement of Vehicles

Block chain applied sciences are now mainly industry driven, lending the problem a barely one-of-a-kind mind-set than other areas of research: With Bitcoin and variations being developed with the aid of practitioners as a replacement than cryptographers, the have self assurance tends to be put now not in formal proofs and houses on the other hand in realistic resistance to assaults principally primarily based on everyday

knowledge and journey thru practitioners ${ }^{[13]}$. Bit coin has now no longer been

immune from confusion over pseudonymity, privateness and anonymity. Bitcoin gives completely 
pseudonymous transactions ${ }^{[15]}$. As Bitcoin is a shared world public ledger, pseudonyms may be additionally de-anonymized with the useful resource of identifying patterns of usage in block chain ${ }^{[6]}$.

Whether self-driving motors improve public health, decrease site visitors congestion, and reduce local weather trade will rely on informed, science-based policy ${ }^{[14]}$. This will require a strong lookup agenda and on hand records on the overall performance and operation of self-driving cars. Vehicle computer systems have to be impervious from hacking, and rigorous testing and regulatory oversight of car programming are vital to make certain that self-driving cars shield each their occupants and those outdoor the automobile [7].

Daimler Carried out a check run in which vehicles made computing device to machine repayments the usage of block chain platform besides any human interaction ${ }^{[16]}$.

The mixture of Autonomous Vehicles and Block chain science has the potential of establishing a secured, relied on a machine where the self reliant car will supply the quit user the most handy route primarily based on real-time site visitors information, and Block chain will make the monetary transaction simpler ${ }^{[8]}$. Crypto Bit Trust that will assist to improve the privateness of Intelligent Vehicle. Trust bit supply fast and impenetrable conversation between Intelligent Vehicles. It also helps to notice the records of Intelligent Vehicle ${ }^{[9]}$.

The purposes of blockchain in a variety of fields, systematically analyzes the protection of every layer of the blockchain and feasible cyber attacks ${ }^{[10]}$. The qualities in the improvement of wise unmanned self enough structures with regard to seven aspects ${ }^{[11]}$.

A decentralized non-public information management gadget that ensures customers own and manage their data. A protocol that turns a blockchain into an automated access-control supervisor that does now not require have confidence in a third party is proposed and implemented $^{[12]}$.

\section{PROPOSED WORK}

Autonomous motors are posted to viable malicious attackers at some point of a number of channels. There are two kinds of inclined channels that would be attacked, inter-communication channels and intra-communication channels. Inter-communication channels encompass the verbal alternate between outdoor archives delivery sites. It consists of visitors information, navigation routing archives and far off firmware enhancements by auto makers.

Another channel is the Inter-communication channel. An self reliant car has the intra-network, which consists of the (ECU), (BCU) and (WCU). The Intra-communication channel wants immoderate safety and security. Ironically, to tightly closed the safety of this intra-communication unit, this regional constantly be related with the auto maker manipulate core to check the attention of the self reliant car, which then will extend the possibility of a cyber-attack through the use of malicious attackers. Here comes the use of block chain.

Block chain is an all encompassing technological know-how that is integrating across systems and hardware all over the world. A block chain is a information shape that makes it viable to create a digital ledger of statistics and share it amongst a network of independent events (Fig.2).

The value and benefits of self-driving motors are nevertheless commonly hypothetical. More records is wished to definitely get right of entry to how they shall influence drivers, the safety, fairness and environmental.

Safety - It is an over arching concern. Lots of human beings die in motor crashes every and each yr in the world; self the use of vehicles could, hypothetically, limit that quantity software program software ought to prove to be much less error - inclined than human beings but cyber protection is a chief difficulty.

Equity - It is every other most important consideration. Self driving technological know-how could assist mobilize men and women who are unable to power themselves, such as the aged or disabled. But the tremendous adaptation of self sufficient cars may want to additionally displace hundreds of thousands of Americans employed as drivers, negatively have an effect on public transportation funding and perpetuate the current day transportation system's injustices. Environmental - Impacts are a serious challenge and predominant uncertainty, accessible, lower priced and convenient self-driving automobiles ought to make bigger the complete variety of miles driven each and each and every yr. If these cars are powered by the use of gasoline, then transportation associated nearby local weather emissions may additionally desire to skyrocket. If however, the motors are electrified and paired with a easy electrical energy grid then transportation emissions have to drop, perhaps highly. Safety and Security - As vehicles end up increasingly self sustaining and self driving automobiles move from the test market to Main Street, protection will grow to be the primary venture for automakers and other stake holders. The greater connected a car is, the extra susceptible it becomes to probably lethal cyber attacks.

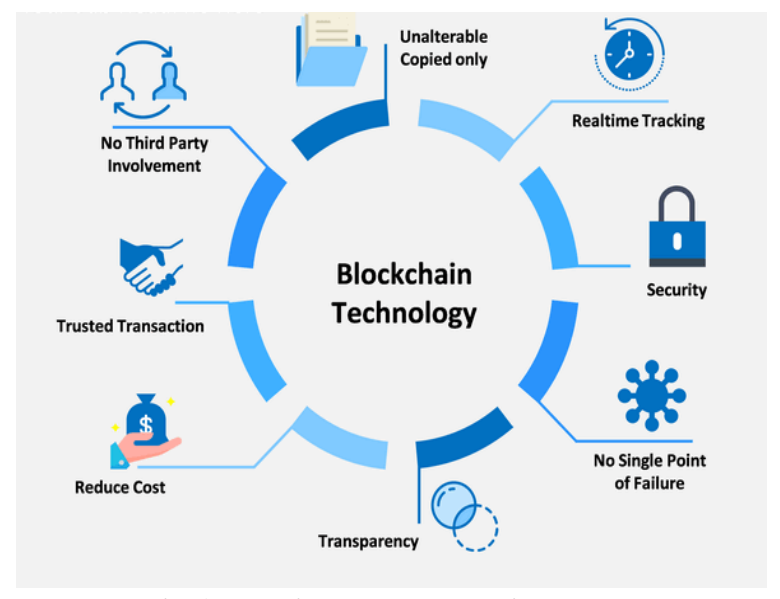

Fig.2: Various Block chain usages

If information streams are to be kept protected from hackers, they have to protect with the absolute best stage of security accessible and that would be block chain. Distributed ledger technological know-how no longer only facilitates facts storage, however additionally affords immutability or protection in opposition to data being changed once it is saved in the block. 
Telematics - Telematics is a mixture of telecommunications and informatics, in the autonomous vehicle space, telematics include software program based totally navigation, vehicle to vehicle $(\mathrm{V} 2 \mathrm{~V})$ communications and a host of different offerings that can have an effect on automobile security and passenger security. The information sent and acquired by using telematics gadget should be stored safe, as you have seen, block chain is ideally suited for simply such applications.

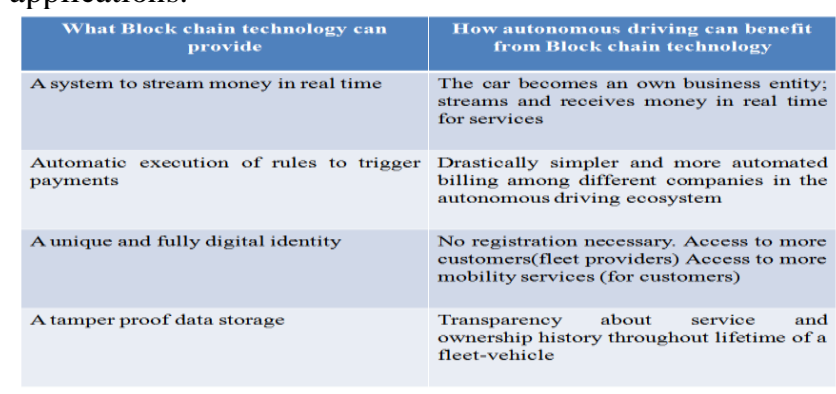

Fig.3: Usage of Block chain in Autonomous Vehicle

Let us mention down other uses of block chain except crypto currency, they are:

First and basic, block chain technology is and also will be highly used for storing all your personal information and you get to decide whi has the access to it and who not.

The use of technology helps us store data, manage data and also share it the concept of smart contracts of block chain technology, also has real amazing uses other than crypto currency, that is the smart contract will be used to design a fully automated system for the industry of supply chain management.

The tasks of the supply chain like assiging, sharing, linking and many more will be linked and integrated with the technology of block chain for the better purpose and services.

Another functional use of block chain technology except crypto currency is also that, block chain technology eliminates the need of middle man, when dealing with others, sharing data, making payments or transactions etc.

By storing data on a block chain, digital certificates for data including car identity, ownership, warranties and mileage can be securely stored in an digital wallet. This record is saved on the block chain and cryptographically verified. The automobile can be accessed only via the permissioned parties (Fig.3).

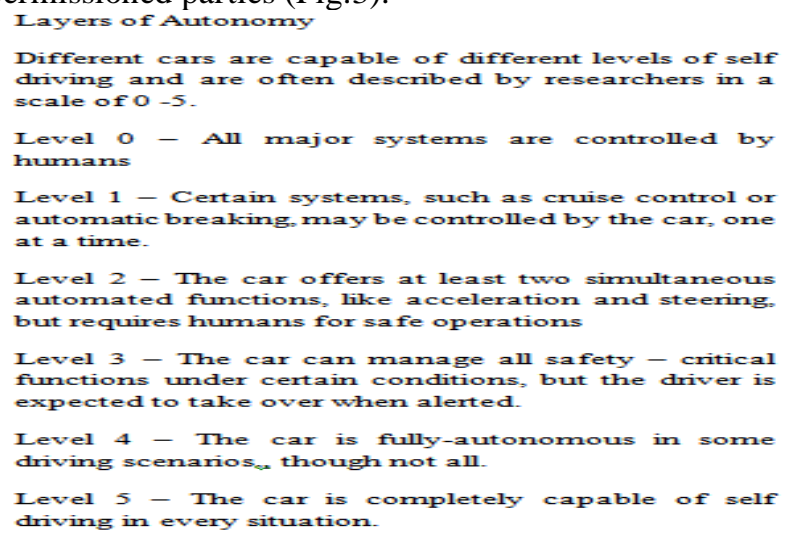

Fig.4: Layers of Autonomy

We create a block with the information of the end user and submit the block in the block chain. Once the block is added it will become public and every one can view it.
Once the block is added to the chain the deletion or hacking of the block is impossible so the data will be secure.

\section{CONCLUSION AND FUTURE WORK}

In this paper we have mentioned the approach in which how Block chain can be used in the independent vehicle in personalizing the vehicle and the method by using which we can invulnerable the data from hackers. Regarding the future work we will be working in the direction how the autonomous vehicle can be transferred to another user with proper authentication.

\section{ACKNOWLEDGMENT}

The authors would like to thank the Department of Information Technology of Kalasalingam Academy of Research and Education, Tamilnadu, India for permitting to use the computational facilities available in open source research laboratory.

\section{REFERENCES}

1. Mohamed Baza et al. "Blockchain-based Firmware Update Scheme Tailored for Autonomous Vehicles" arXiv:1811.05905v1 [cs.CY] 14 Nov 2018

2. Matevz Pustisek et al. "Blockchain based Autonomous Selection of Electric Vehicle Charging Station" International Conference on Identification, Information and knowledge in the Internet of Things(IIKI) 05 Feb 2016

3. Emy Rivera et al. "A Study of Unmanned Vehicles and Cyber Security"

4. Giulio Malavolta et al. "Anonymous Multi-Hop Locks for Blockchain Scalability and Interoperability" NDSS Feb 2019

5. Mathieu Chanson et al. "Privacy-Preserving Data Certification inthe Internet of Things: Leveraging Blockchain Technology to Protect Sensor Data" Journal Article 2019"6. Introduction to Security and Privacy on the Blockchain"

6. Harry Halpin, Marta Piekarska "Introduction to Security and Privacy on the Blockchain" 2017 IEEE Eutopean Symposium on Security and Privacy Workshop.

7. Maximizing the Benefits of Self-Driving Vehicles Union of Concerned Scientists www.ucsusa.org/avprinciples.

8. Panagiota Georgia Saranti et al. "Autonomous Vehicles and Blockchain technology are shaping the future of Transportation" May 2018

9. Madhusudan Singh, Shiho Kim, "Blockchain based Intelligent Vehicle Data Sharing Framework", arXiv preprint, arXiv:1708.09721, 01 Sept. 2017

10. Hai Wang et al. "An Overview of Block Chan Security Analysis" CNCERT 2018 https://doi.org/10.1007/978-981-13-6621-5 5

11. Tao ZHANG et al. "Current trends in the development of intelligent unmanned autonomous systems" http://dx.doi.org/10.1631/FITEE.1601650

12. Guy Zyskind, Oz Nathan, Alex 'Sandy' Pentland "Decentralizing Privacy: Using Blockchain to Protect Personal Data"

13. Boohyung Lee, Jong-Hyouk Lee "Blockchain-based secure firmware update for embedded devices in an Internet of Things environment" doi: 10.1007/s11227-016-1870-0 Cross Mark

14. Oded Leiba et al. "Incentivized Delivery Network of IoT Software Updates Based on Trustless Proof -of-Distribution" IEEE Workshop on Security \& Privacy on the Blockchain

15. Noam Copel, Tal Ater "Decentralized Autonomous Vehicle While Paper"

16. https://cointelegraph.com/news/can-blockchain-become-an-integral-partof-autonomous-vehicles. 


\section{AUTHORS PROFILE}

Prem Raja he is a working as a Assistant professor and a Part-Time research scholar in the department of Information Technology at Kalasalingam Academy of Research and Education, Krishnankoil, Srivilliputhur India. His areas of interest are big data analytics, autonomous vehicles and security.

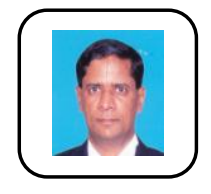

Vasudevan he is working as senior professor in the department of information technology of Kalasalingam Academy of Research and Education Krishnankoil Srivilliputhur India for past 27 years and his areas of interest are big data analytics, cloud computing, network security and block chain technology. He has published 170 above papers in international journals and conferences. He has produced twenty above $\mathrm{PhD}$ scholars and currently guiding five $\mathrm{PhD}$ scholars. He is a life time member in ISTE and IAENG. He received Dr. APJ Abdul Kalam Award for life time contribution in teaching on 2016 . 